\title{
Sozial arbeiten im sozialwirtschaftlichen Horizont
}

\section{Gutes tun und Güter erzeugen}

\author{
Wolf Rainer Wendt
}

\begin{abstract}
In bewegten Verhältnissen müssen Akteure ibre Position immer wieder neu bestimmen. Das gilt auch und insbesondere für die Soziale Arbeit. Ihre Identität, ihr Selbstverständnis und ibre Einordnung in Leistungsprozesse, die der Sozialstaat vorsieht und die der gesellschaftliche Wandel fordert, stehen zur Disposition. Zwei Neuerscheinungen geben Anlass zu einer aktuellen Bestandsaufnahme.
\end{abstract}

Zur sozialberuflichen Orientierung tragen Publikationen bei, die vielseitige Wissensbestände und Begriffsbestimmungen versammeln. Sehen wir einmal nach, wie zwei gewichtige Nachschlagewerke, die kürzlich erschienen sind, die Soziale Arbeit verorten:

- Das eine ist das »Fachlexikon der sozialen Arbeit «. Es gehört zu den am häufigsten gebrauchten Büchern im Sozialwesen und hat seinen inhaltlichen Schwerpunkt im administrativen Bereich. Soziale Arbeit wird im Feld ihrer Betätigung beschrieben und an den einzelnen Stellen ihres Vorkommens vornehmlich unter institutionellen und funktionalen Gesichtspunkten behandelt.

- Für die dringliche ökonomische Erörterung, wozu Soziale Arbeit gebraucht wird und was sie »bringt «, schauen wir in das neue "Lexikon der Sozialwirtschaft «. Sein Schwerpunkt liegt im Management, in der Führung der Geschäfte von sozialen Unternehmen. Ihre Geschäfte sind in Bewegung und mit ihnen die Anforderungen an das Handeln in ihnen. Wie verhält sich Soziale Arbeit zu diesem »Business«? Das

Prof. Dr. Wolf Rainer Wendt ist Vorsitzender der Deutschen Gesellschaft für Soziale Arbeit e. V. Internet http://www.deutschegesellschaft-fuer-soziale-arbeit.de
Handbuch beantwortet diese Frage nicht. Die sozialprofessionelle Befassung mit der Sozialwirtschaft wirft sie aber auf.

In den Diensten und Einrichtungen des sozialwirtschaftlichen Sektors gehen Sozialberufler ihrer Beschäftigung an vielen Stellen und in verschiedenen Aufgabenfeldern nach. Sie haben im täglichen personenbezogenen Einsatz kaum Einfluss auf das Arrangement der sozialwirtschaftlichen Angebote und auf ihren Betrieb. Über dessen Gestaltung wird auf der Organisationsebene und sozialpolitisch entschieden. Zur Betriebsführung gehört auch die Entscheidung im Personalwesen, inwieweit Sozialarbeiterinnen und Sozialarbeiter beschäftigt werden oder andere mehr oder minder qualifizierte Kräfte zur Erledigung von Aufgaben herangezogen werden. Es gibt keine selbstverständliche Übereinkunft, dass dort, wo man sozial wirtschaftet, sozialberuflich gearbeitet wird.

\section{Wohlfahrt leisten}

In den einschlägigen Diensten und Einrichtungen wird gepflegt, betreut, erzogen, behandelt, beraten, rehabilitiert und integriert. Kurz: Es wird auf vielfältige Art eine Versorgung geleistet. Das Sachziel insgesamt ist individuelles und gemeinschaftliches Wohlergehen. Daran an der »Wohlfahrt « - wird gearbeitet. Ein soziales Unternehmen ist dem Ergehen von Menschen zuträglich und zwar nicht einer bestimmten einzelnen Person, vielmehr Personengruppen, die in Hinblick auf Pflege, Betreuung, Erziehung, Behandlung, Beratung, Rehabilitation und Teilhabe bedürftig sind. Die Aufgabe wird »sozial« erledigt, das heißt, Menschen arbeiten mit Menschen und man kommt dabei gemeinsamen oder gemeinschaftsbezogenen Erfordernissen nach, wenngleich im direkten personenbezogenen Einsatz ein individuelles Problem be- handelt und einem diskreten persönlichen Bedarf abgeholfen wird.

Berufliche Soziale Arbeit ist dabei nicht unabdingbar. Sozialarbeiterinnen und Sozialarbeiter finden sich im Beschäftigungssystem in Konkurrenz zu anderen Humanberufen und haben zudem Schwierigkeiten, im eigenen sozialprofessionellen Handlungsbereich zu definieren, wer beruflich zu ihm gehört oder ihm nicht zugerechnet werden sollte: Sozialpädagogen, Diplompädagogen, Heilpädagogen, Erzieher, Supervisoren und diverse Beratungskräfte. Hinzu kommt der Klärungsbedarf im Verhältnis zu dem Personal, das in den Organisationen und Diensten der Sozialen Arbeit mitwirkt, oft in leitender Position, ohne sozialprofessionell ausgebildet $\mathrm{zu}$ sein, und die Notwendigkeit, sich zu den freiwillig und bürgerschaftlich Engagierten im Handlungsfeld in ein Verhältnis zu setzen.

Im »Lexikon der Sozialwirtschaft «, das die Aktivitäten, Formen und Institutionen behandelt, in und mit denen zu einem sozialen Zweck gearbeitet wird, finden wir die Soziale Arbeit explizit nur in den Artikeln "Sozialarbeit/Sozialpädagogik « und "Sozialarbeiter - Sozialpädagoge« erörtert. Das Verhältnis der Sozialen Arbeit zur Sozialwirtschaft oder auch zur »Wohlfahrtspflege « insgesamt wird in den beiden Artikeln und auch anderswo nicht eigens thematisiert. Es gibt zudem im Lexikon kein Stichwort »Wohlfahrtspflege «, in dem sich das sozialprofessionelle Handeln betrieblich hätte auslegen lassen. Nämlich ob es mit der »Pflege« von Wohlfahrt gleichzusetzen oder wie es in ihr einzugrenzen ist. Leistet ein Sozialprofessioneller sie - oder trägt er nur in bestimmter Weise zum Wohlfahrtsziel bei? Sich mit dieser Frage zu befassen, war nicht Aufgabe derjenigen, die zum »Lexikon der Sozialwirtschaft « beitrugen. Aber sie stellt sich.

Die Klärung des Verhältnisses von Sozialer Arbeit zur Sozialwirtschaft hängt an der Entscheidung, wie begrifflich eng 
oder weit man Soziale Arbeit fassen will. Man mag mit ihr den ganzen Prozess der Leistungserstellung im sozialwirtschaftlichen Betrieb bezeichnen oder eine Komponente, ein » Einsatzgut « neben anderen humandienstlichen Beiträgen. Bedenken wir auch, dass die Menschen, um deren Probleme es im personenbezogenen Dienst geht, mit ihrem Verhalten den Erfolg oder Misserfolg unabdingbar mitbe-
Betrieb. Zu erörtern wäre der Produktionsfaktor Soziale Arbeit in Hinblick auf den erwarteten und erzielten Güterertrag.

Für die berufliche Praxis ist die Methodenkompetenz zentral. Sozialarbeiterinnen und Sozialarbeiter wissen, welche Wege zur Problembewältigung beschritten werden können. Die dabei zu treffenden Dispositionen müssen indes in Hinblick auf Bedarf, Ressourcen, Kosten und

\section{»Berufliche Sozialarbeit schließt an persönliche und familiäre Sorgearbeit an «}

stimmen, so halten wir uns mit der Frage der richtigen Faktorkombination bereits mitten in der Mikroökonomie der sozialen Wohlfahrtsproduktion auf.

In der sozialwirtschaftlichen Produktion stellt das Management den dispositiven Faktor dar, der für Leitung, Organisation, Planung und Kontrolle eingesetzt wird. Der Elementarfaktor des personenbezogenen sozialen Arbeitseinsatzes wird gebraucht, um einzelne Ziele des Dienstbetriebs erst mit seinen Nutzern auszumachen und dann methodisch zu erreichen. Hat das Management Entscheidungen darüber getroffen, an welcher Stelle und in welchem Ausmaß Mitarbeiter eingesetzt werden, trifft die einzelne Fachkraft ihrerseits Allokationsentscheidungen, indem sie ihre Zeit und Kraft verteilt und zusammen mit den Adressaten ihrer Bemühungen verfügbare immaterielle und materielle Mittel bestimmten Zwecken zuweist. Insoweit handeln Sozialarbeiterinnen und Sozialarbeiter gewöhnlich managerial.

Im »Lexikon der Sozialwirtschaft " wird unter dem Stichwort "Sozialarbeit/Sozialpädagogik « die professionelle Praxis unter fachlichen und konzeptuellen Gesichtspunkten abgehandelt, nicht unter dem Aspekt einer ökonomischen Leistungserbringung. "Sozialarbeiter/Sozialpädagogen « üben - ihrem Selbstverständnis nach - eine Tätigkeit aus, die berufsethisch und wissenschaftlich fundiert und universellen Menschenrechten verpflichtet ist, heißt es im nachfolgenden Artikel. Offen bleibt die Einordnung dieser Tätigkeit in den sozialwirtschaftlichen
Nutzen gerechtfertigt sein. Die Güte der Arbeitsverrichtungen allein, die Angemessenheit der Methode mag fachlich für die Prozessqualität stehen, für die InputOutput-Relation ist sie kein hinreichendes Kriterium. Man muss Prioritäten setzen, Mitwirkung einschätzen, Nebenfolgen einkalkulieren usw. Das Integral der Methode ist ein Wirtschaften.

\section{Sorgen und Versorgung}

Soziales Wirtschaften integriert auf der Mikroebene die Versorgungsleistungen der in einem Dienst oder einer Einrichtung Beschäftigten und die Eigenaktivität der Menschen, für die etwas getan wird. Berufliche Sozialarbeit schließt an persönliche und familiäre Sorgearbeit an. Außerdem kommt zum professionellen Handeln informelle freiwillige Mitarbeit. Die Betätigungen sind ökosozial eingebunden in Vorgänge, für die als leitende Begriffe ein haushaltendes Handeln, Ressourcenerschließung, gemeinschaftliche und individuelle Lebensgestaltung, Selbstorganisation und Kompetenzentwicklung, sozialräumliche Einbettung, Integrierte Versorgung genannt werden können. Hierfür angemessene Arrangements zu erreichen und zu unterhalten, ist die manageriale Aufgabe, die auf vielen Gebieten professioneller Sozialer Arbeit erledigt werden muss.

Macht sich eine Sozialarbeiterin beruflich selbstständig, so führt sie einen Einpersonenbetrieb, der die Sorge von problembelasteten Menschen zum Anlass für eine mit ihnen zu vereinbarende Versorgung nimmt. Der Arbeitseinsatz der Fachkraft schließt die Bewirtschaftung der Aufgabe und die Gestaltung des Arbeitseinsatzes ein. Hier wird das sozialwirtschaftliche Format der Berufsarbeit deutlich. Sozialprofessionelle sind Akteure im sozialwirtschaftlichen Horizont und sollten sich in ihm mit all seinen zivilen, rechtlichen, finanziellen, politischen und administrativen Beziehungen zwischen Leistungsnehmern, Leistungserbringern und Leistungsträgern umtun.

Deutscher Verein für öffentliche und private Fürsorge e. V. (Hg.): Fachlexikon der sozialen Arbeit. 6. Auflage. Nomos Verlagsgesellschaft, Baden-Baden 2007, 1.195 Seiten. 44,- Euro (Vorzugspreis für Mitglieder des Deutschen Vereins und Studenten 34,Euro). ISBN 978-3-8329-1825-5.

Bernd Maelicke (Hg.): Lexikon der Sozialwirtschaft. Nomos Verlagsgesellschaft, Baden-Baden 2007. 1.128 Seiten. 98,- Euro. ISBN 978-3-8329-2511-6.

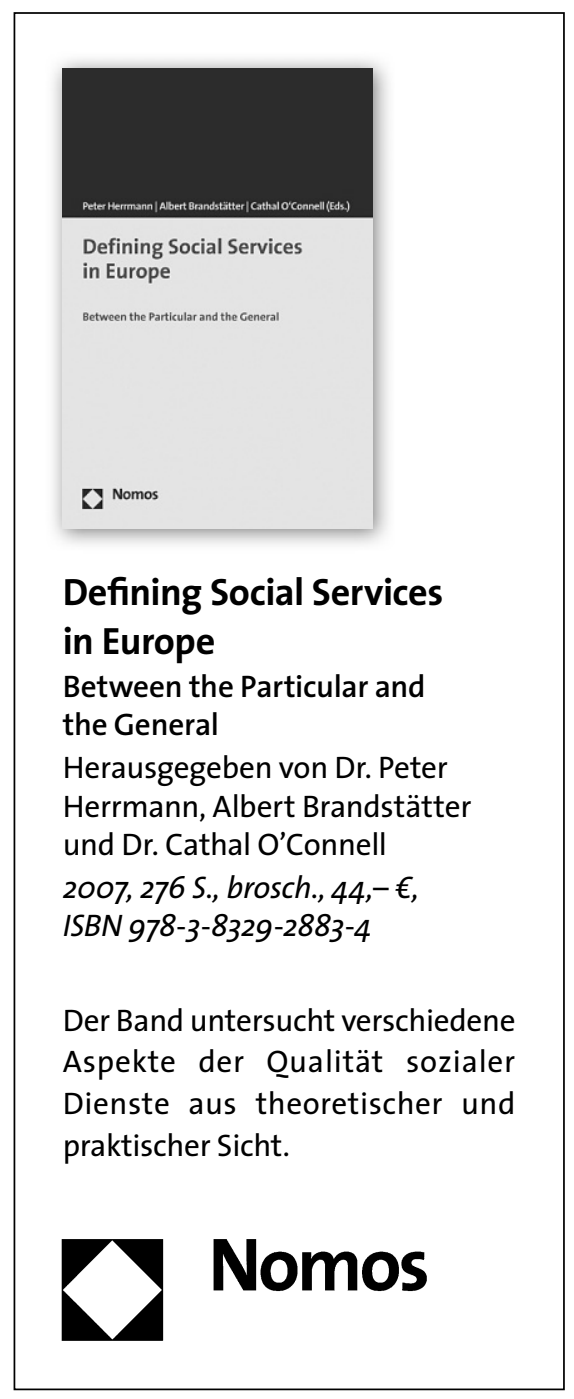

